\title{
Further results on the WIMP annual modulation signature by DAMA/Nal
}

\author{
Rita Bernabei, Pierluigi Belli*, Fabio Cappella, Francesco Montecchiał Francesco \\ Nozzoli \\ Dip. di Fisica, Universita' di Roma "Tor Vergata" \\ and INFN, sez. Roma2, I-00133 Rome, Italy \\ E-mail: pierluigi.beliọroma2.infn.iti
}

\section{Antonella Incicchitti, Daniele Prosperi}

Dip. di Fisica, Universita' di Roma "La Sapienza"

and INFN, sez. Roma, I-00185 Rome, Italy

\section{Riccardo Cerulli}

INFN - Laboratori Nazionali del Gran Sasso, I-67010 Assergi (Aq), Italy

C.J. Dai, H.H. Kuang, J.M. Ma, Z.P. Ye

IHEP, Chinese Academy, P.O. Box 918/3, Beijing 100039, China

ABSTRACT: The main aim of the $\simeq 100 \mathrm{~kg}$ highly radiopure NaI(Tl) set-up of the DAMA project (DAMA/NaI) has been the exploitation of the model independent WIMP annual modulation signature. The total exposure $(107731 \mathrm{~kg} \times$ day), collected during seven annual cycles, has given a model independent evidence for the presence of a Dark Matter particle component in the galactic halo at $6.3 \sigma$ C.L.; this main result is summarised here. Some of the many possible corollary model dependent quests for the candidate particle are also mentioned. At present, after about five years of new developments, a second generation low background set-up (DAMA/LIBRA with a mass of $\simeq 250 \mathrm{~kg} \mathrm{NaI}(\mathrm{Tl})$ ) was built and is taking data since March 2003. New R\&D efforts toward a possible $\mathrm{NaI}(\mathrm{Tl})$ ton set-up, we proposed in 1996, have been funded and started in 2003.

\section{Introduction}

The DAMA project has been proposed by the italian group to INFN and firstly funded in $1990[1 \overline{1}]$; in 1992 the chinese colleagues joined the project. DAMA is an observatory for

${ }^{*}$ Speaker.

†also: Universita' "Campus Bio-Medico" di Roma, 00155, Rome, Italy

${ }^{\ddagger}$ also: University of Zhao Qing, Guang Dong, China 
rare processes based on the development and use of various kinds of radiopure scintillators. Several low background set-ups have been realised; the main ones are: i) DAMA/NaI $(\simeq 100 \mathrm{~kg}$ of radiopure $\mathrm{NaI}(\mathrm{Tl})$ ), which took data underground over seven annual cycles and was put out of operation in July 2002; ii) DAMA/LXe ( $\simeq 6.5 \mathrm{~kg}$ liquid Xenon); iii) DAMA/R\&D, which is devoted to tests on prototypes and to small-scale-experiments; iv) the new second generation set-up DAMA/LIBRA ( $\simeq 250 \mathrm{~kg}$; more radiopure $\mathrm{NaI}(\mathrm{Tl})$ ) in operation since March 2003. Moreover, in the framework of devoted R\&D for higher radiopure detectors and PMTs, sample measurements are regularly carried out by means of the low background DAMA/Ge detector, installed deep underground since $\gtrsim 10$ years and, in some cases, by means of Ispra facilities. Some recent results from DAMA/LXe and

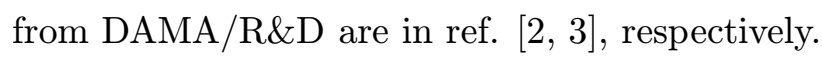

The DAMA/NaI set-up and its performances have been described in details in ref. [i]; since then some upgrading has been carried out [i5in]. The first data release and publication of DAMA/NaI data occurred in $1996[6]$ [י] analysed by using the pulse shape discrimination (PSD) technique in $\mathrm{NaI}(\mathrm{Tl})$. Investigation of possible diurnal effects has been carried out as well [i] $[\bar{z}]$. Moreover, DAMA/NaI has also obtained results on the investigation of several other topics and other rare processes [i్

The main aim of the experiment was actually the investigation of the presence of a Dark Matter particle component in the galactic halo by means of the model independent WIMP annual modulation signature, which has been deeply investigated over seven annual cycles $(107731 \mathrm{~kg} \times$ day total exposure). The obtained results - whose details can be found

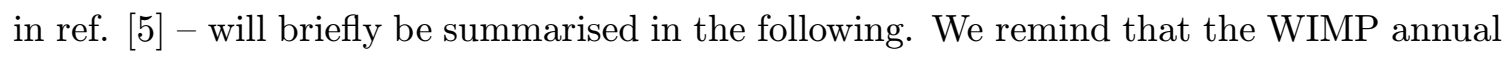
modulation signature is based on the annual modulation of the signal rate induced by the Earth revolution around the Sun; as a consequence, the Earth will be crossed by a larger WIMP flux roughly in June (when its rotational velocity is summed to the one of the solar system with respect to the Galaxy) and by a smaller one roughly in December (when the two velocities are subtracted). The annual modulation signature [īj] is very distinctive since a WIMP-induced seasonal effect must simultaneously satisfy all the following requirements: the rate must contain a component modulated according to a cosine function (1) with one year period, $T,(2)$ and a phase, $t_{0}$, that peaks roughly around $\simeq 2^{\text {nd }}$ June (3); this modulation must only be found in a well-defined low energy range, where WIMP induced recoils can be present (4); it must apply to those events in which just one detector of many actually "fires" (single-hit events), since the WIMP multi-scattering probability is negligible (5); the modulation amplitude in the region of maximal sensitivity is expected to be $\lesssim 7 \%$ (6) with the exception of some possible scenarios such as e.g. those in refs.

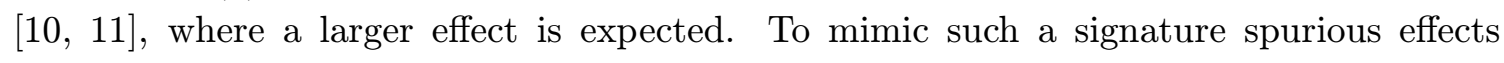
or side reactions should not only be able to account for the whole observed modulation amplitude, but also to contemporaneously satisfy all the requirements; no one has been found or suggested by anyone over about a decade.

The presence of a model independent positive evidence in the data of DAMA/NaI has been firstly reported by the DAMA collaboration at the TAUP conference in 1997

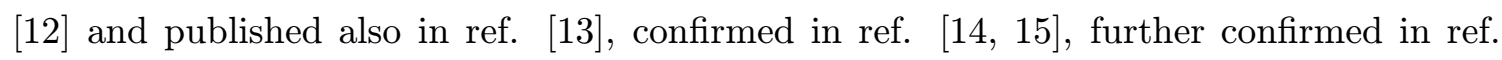

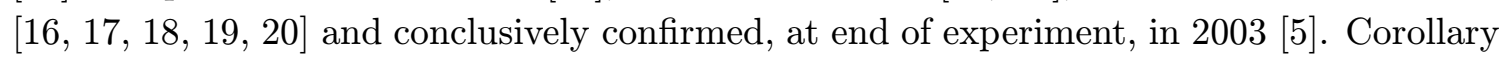


model dependent quests for a candidate particle have been carried out in some of the many possible model frameworks and have been improved with time. In particular, some scenarios either for mixed spin-independent (SI) and spin-dependent (SD) coupled WIMPs or for purely SI coupled WIMPs or for purely SD ${ }^{1}$ coupled WIMPs have been considered in some of the many possible model frameworks as well as the case of WIMPs with preferred inelastic scattering.

Note that some comments on the wrong/arbitrary claims for contradiction by Cdms-I,

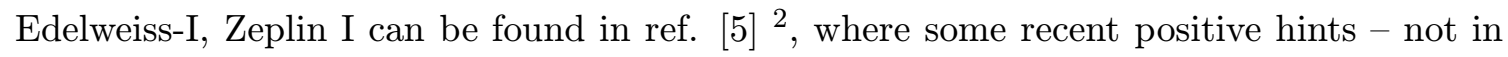
contradiction with the DAMA/NaI result - from Dark Matter indirect searches are also summarised.

The new second generation larger mass and higher radiopure $\mathrm{NaI}(\mathrm{Tl})$ set-up, named DAMA/LIBRA, is now in operation and will be briefly introduced.

\section{Final DAMA/NaI model independent result on WIMP annual modu- lation signature over 7 annual cycles}

A model independent approach on the data of the seven annual cycles (total exposure: $107731 \mathrm{~kg} \times$ day) offers an immediate evidence of the presence of an annual modulation of the rate of the single-hit events in the lowest energy region as shown in Fig. 竞- left (for more see ref. $\left.\cos \omega\left(t-t_{0}\right)\right)$ at $6.3 \sigma$ C.L. and their fit for the $(2-6) \mathrm{keV}$ larger statistics energy interval offers modulation amplitude equal to $(0.0200 \pm 0.0032) \mathrm{cpd} / \mathrm{kg} / \mathrm{keV}, t_{0}=(140 \pm 22)$ days and $T=\frac{2 \pi}{\omega}=(1.00 \pm 0.01)$ year, all parameters kept free in the fit. The period and phase agree with those expected in the case of a WIMP induced effect ( $T=1$ year and $t_{0}$ roughly at $\simeq 152.5^{\text {th }}$ day of the year). The $\chi^{2}$ test on the $(2-6) \mathrm{keV}$ residual rate disfavours the hypothesis of unmodulated behaviour giving a probability of $7 \cdot 10^{-4}\left(\chi^{2} /\right.$ d.o.f. $\left.=71 / 37\right)$. The same data have also been investigated by a Fourier analysis (performed according to

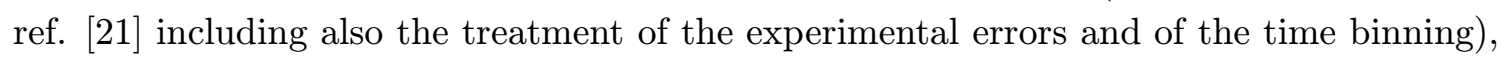
obtaining the result shown in Fig. $i_{-1}^{1}-$ right, where a clear peak corresponding to a period of 1 year is evident.

In Fig. $\overline{2}_{i}$ the experimental single-hit residual rate from the total exposure of 107731 $\mathrm{kg} \times$ day is presented, as in a single annual cycle, for two different energy intervals; as it can be seen the modulation is clearly present in the $(2-6) \mathrm{keV}$ energy region, while it is absent just above.

\footnotetext{
${ }^{1}$ We remind that JHEP 0107 (2001) 044 is not at all in conflict with a purely SD solution since it considered only two particular purely SD couplings (of the many possible) in a strongly model dependent context and using modulation amplitudes valid instead only in a particular purely SI case. Moreover, the mixed SI \& SD case was not involved at all in that discussion.

${ }^{2}$ Here we just remind e.g. that no model independent comparison is possible among those experiments and DAMA/NaI because of the different methodological approaches, of the different target nuclei, etc.. As regards possible model dependent comparisons, those experiments give result in a single purely SI model framework with fixed/selected assumptions, neglecting experimental and theoretical uncertainties and ignoring the existence of other solutions; in addition, they have quoted so far the DAMA/NaI result in an incorrect and incomplete way.
} 

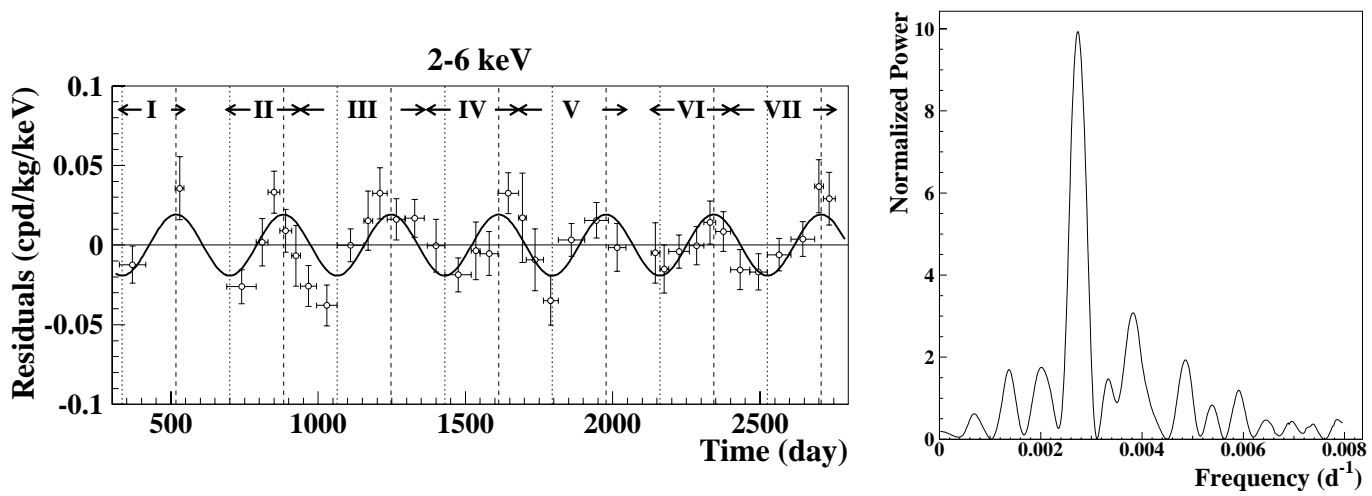

Figure 1: On the left: experimental residual rate for single-hit events in the (2-6) keV energy interval as a function of the time over 7 annual cycles (total exposure $107731 \mathrm{~kg} \times$ day); end of data taking July 2002. The experimental points present the errors as vertical bars and the associated time bin width as horizontal bars. The superimposed curve represents the cosinusoidal function behaviour expected for a WIMP signal with a period equal to 1 year and phase exactly at $2^{\text {nd }}$ June; the modulation amplitude has been obtained by best fit. See ref. [inj]. On the right: power spectrum of the measured (2-6) $\mathrm{keV}$ single-hit residuals calculated including also the treatment of the experimental errors and of the time binning. As it can be seen, the principal mode corresponds to a frequency of $2.737 \cdot 10^{-3} \mathrm{~d}^{-1}$, that is to a period of $\simeq 1$ year.
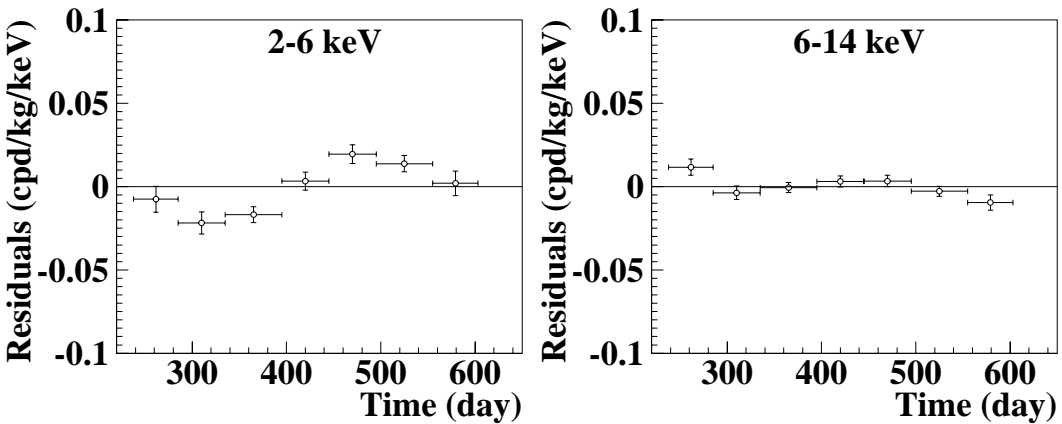

Figure 2: Experimental single-hit residual rate from the total exposure of $107731 \mathrm{~kg} \times$ day as in a single annual cycle in the (2-6) keV energy interval (on the left) and in the (6-14) keV energy interval (on the right). The experimental points present the errors as vertical bars and the associated time bin width as horizontal bars. The initial time is taken at August $7^{\text {th }}$. Fitting the data with a cosinusoidal function with period of 1 year and phase at 152.5 days, the following amplitudes are obtained: $(0.0195 \pm 0.0031) \mathrm{cpd} / \mathrm{kg} / \mathrm{keV}$ and $-(0.0009 \pm 0.0019) \mathrm{cpd} / \mathrm{kg} / \mathrm{keV}$, respectively. Thus, a clear modulation is present in the lowest energy region, while it is absent just above.

Finally, a suitable statistical analysis has shown that the modulation amplitudes are statistically well distributed in all the crystals, in all the data taking periods and considered

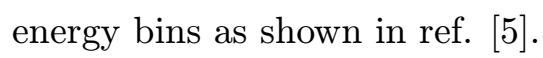

A careful investigation of all the known possible sources of systematics and side reactions has been regularly carried out and published at time of each data release where

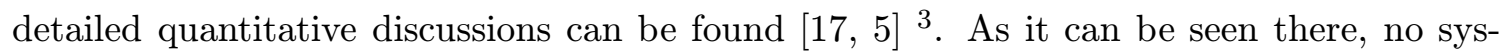

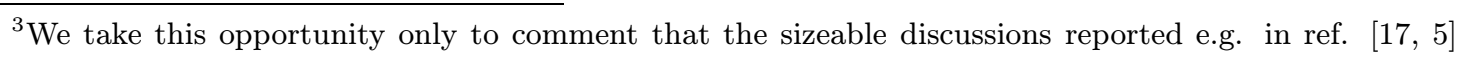
already demonstrated that a possible modulation of neutron flux (possibly observed by the ICARUS coll. as reported in the ICARUS internal report TM03-01) cannot quantitatively contribute to the DAMA/NaI observed modulation amplitude, even if the neutron flux would be assumed to be 100 times larger than
} 
tematic effect or side reaction able to mimic a WIMP induced effect has been found.

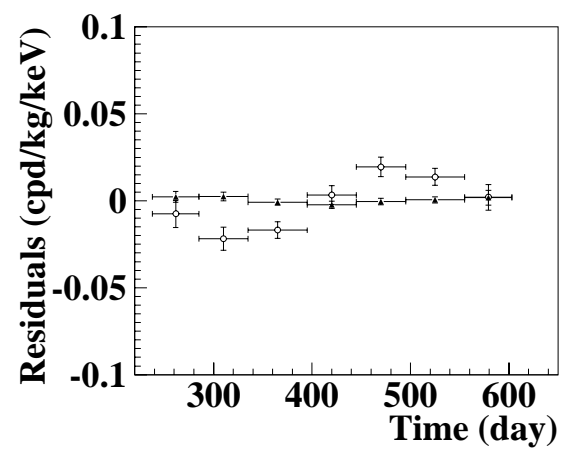

Figure 3: Experimental residual rates in the (2-6) keV cumulative energy interval for singlehit events (open circles) - class of events to which WIMP events belong - over seven annual cycles and for multiple-hits events (filled triangles) - class of events to which WIMP events do not belong - over the last two annual cycles. They have been obtained by considering for each class of events the data as collected in a single annual cycle and using in both cases the same identical hardware and the same identical software procedures. The initial time is taken on August $7^{\text {th }}$. Thus, while a clear modulation is present in the residual rates of the single-hit events, it is absent in the residual rates of the multiple-hits events. $A=(0.0195 \pm 0.0031) \mathrm{cpd} / \mathrm{kg} / \mathrm{keV}$ and $A=-(3.9 \pm 7.9) \cdot 10^{-4} \mathrm{cpd} / \mathrm{kg} / \mathrm{keV}$ for singlehit and multiple-hits residual rates, respectively. Thus, a $6.3 \sigma$ C.L. evidence of annual modulation is present in the single-hit residuals (events class to which the WIMP-induced recoils belong), while it is absent in the multiple-hits residual rate (event class to which only background events belong). Since the same identical hardware and the same identical software procedures have been used to analyse the two classes of events, the obtained result offers an additional strong support for the presence of Dark Matter particles in the galactic halo further excluding any side effect either from hardware or from software procedures or from background.

Conclusion. In conclusion, the presence of an annual modulation in the single-hit events residual rate in the lowest energy interval $(2-6) \mathrm{keV}$ satisfying all the features expected for a WIMP component in the galactic halo is supported by the data of the seven annual cycles at $6.3 \sigma$ C.L.. This is the experimental result of DAMA/NaI. It is model independent; no other experiment whose result can be directly compared with this one is available so far in the field of Dark Matter investigation.

measured at LNGS by several authors with different techniques over more than 15 years; in addition, as widely known, it cannot satisfy all the peculiarities of the signature mentioned above. 


\section{Corollary result: quests for a candidate particle in some model frame- works with the data of the seven annual cycles}

On the basis of the obtained model independent result, corollary investigations can also be pursued on the nature and coupling of the WIMP candidate. This latter investigation is instead model dependent and - considering the large uncertainties which exist on the astrophysical, nuclear and particle physics assumptions and on the parameters needed in the calculations - has no general meaning (as it is also the case of exclusion plots and of the WIMP parameters evaluated in indirect search experiments). Thus, it should be handled in the most general way as we have preliminarily pointed out with time in the

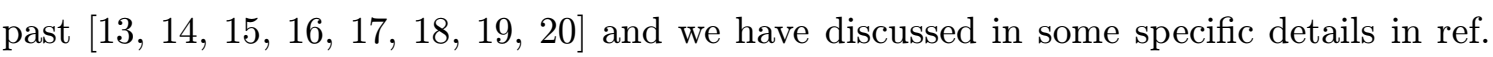
[5]; other efforts on this topic are also in progress. Candidates, kinds of WIMP couplings with ordinary matter and implications, cross sections, nuclear form factors, spin factors,

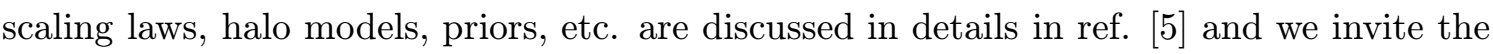
reader to this reference since these arguments are necessary to correctly understand the results obtained in corollary quests and the real validity of any claimed model dependent comparison in the field. The results presented in ref. course, not exhaustive of the many possible scenarios which at present level of knowledge cannot be disentangled. Some of the open questions are: i) which is the right nature for the WIMP particle ${ }^{4}$; ii) which is its right couplings with ordinary matter (mixed SI\&SD, purely SI, purely SD or preferred inelastic) iii) which are the right form factors and related parameters for each target nucleus; iv) which is the right spin factor for each target nucleus (some nuclei are disfavoured to some kinds of interactions; for example, in case of an interaction with SD component even a nucleus sensitive in principle to SD interaction could be blinded by the spin factor if unfavoured by the $\theta$ value $^{5}$ ); v) which are the right scaling laws (let us consider as an example that even in a MSSM framework with purely SI interaction the scenario could be drastically modified as discussed recently in ref. [203i]); vi) which is the right halo model and related parameters; vii) which are the right values of the experimental parameters within their uncertainties; etc. As an example, we remind that not only large differences in the measured rate can be expected when using target nuclei sensitive to the SD component of the interaction (such as e.g. ${ }^{23} \mathrm{Na}$ and ${ }^{127} \mathrm{I}$ )

\footnotetext{
${ }^{4}$ Several candidates fulfil the cosmological and particle Physics requirements necessary in order to be considered as a Dark Matter candidate particle: not only the neutralino foreseen in the supersymmetric theories, but also a heavy neutrino of a $4^{\text {th }}$ family (there is still room for it as reported in literature), the sneutrino in the scenario described by [10] (providing - through the transition from lower to upper mass eigenstate - preferred inelastic scattering with target-nuclei), the "mirror" Dark Matter [22in'], etc.. Moreover, in principle whatever Weakly Interacting, neutral, (quasi-)stable and Massive (whose acronym is WIMP) particle, even not yet foreseen by a theory, can be a suitable candidate. As regards in particular the neutralino, we note that the theories have not stringent predictive capability for its cross sections and for its mass because of the large number of free parameters in the theory and of the several assumptions required; thus, e.g. the expectations for its nuclear cross sections span over several orders of magnitude as it can also be seen in literature. In addition, we take this occasion to remind that the neutralino has both SI and SD couplings with the ordinary matter.

${ }^{5}$ We remind that $\operatorname{tg} \theta=a_{n} / a_{p}$ is the ratio between the WIMP-neutron and the WIMP-proton effective SD coupling strengths, $a_{n}$ and $a_{p}$, respectively [18,
} 
with respect to those largely insensitive to such a coupling (such as e.g. ${ }^{n a t} G e$ and ${ }^{n a t} S i$ ), but also when using different target nuclei although all - in principle - sensitive to such a coupling (compare e.g. the Xenon and Tellurium cases with the Sodium and Iodine cases).

In the following some of the results discussed for some of the many possible model

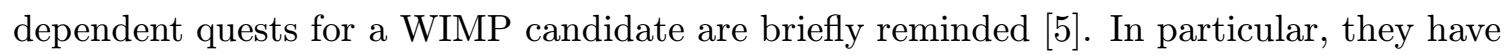
been obtained from the data collected during all the seven annual cycles, considering the

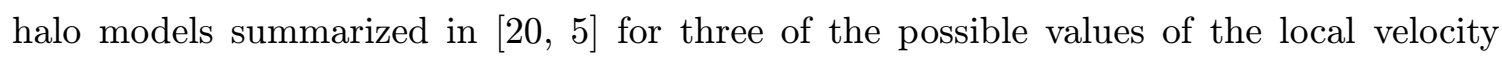
$v_{0}: 170 \mathrm{~km} / \mathrm{s}, 220 \mathrm{~km} / \mathrm{s}$ and $270 \mathrm{~km} / \mathrm{s}$. The escape velocity has been maintained at the fixed value: $650 \mathrm{~km} / \mathrm{s}$. It is worth to note that the present existing uncertainties on the knowledge of the escape velocity can play a relevant role in evaluating allowed regions (and corresponding best fit values for WIMP mass and cross section) e.g. in the cases of preferred inelastic WIMPs and of light mass WIMP candidates; its effect would be instead marginal at large WIMP masses. The possible scenarios have been exploited for those halo models in some discrete cases including some of the uncertainties which exist in the parameters of the used nuclear form factors and in the quenching factors; for the details see ref. [ini]. The results summarised here are not exhaustive of the many scenarios possible at present level of knowledge: e.g. for some other recent ideas see the already quoted [1] $\left.\overline{1}_{1}^{1}, \underline{2}_{2} \overline{3} \overline{3}\right]$.

For simplicity, here the results of these corollary quests for a candidate particle are presented in terms of allowed regions obtained as superposition of the configurations corresponding to likelihood function values distant more than $4 \sigma$ from the null hypothesis (absence of modulation) in each of the several (but still a limited number) of the possible model frameworks considered here. Obviously, larger sensitivities than those reported in the following figures would be reached when including the effect of other existing uncertainties on the astrophysical, nuclear and particle Physics assumptions and related parameters; similarly, the set of the best fit values would also be enlarged as well.

As well known, DAMA/NaI is intrinsically sensitive both to low and high WIMP mass having both a light (the ${ }^{23} \mathrm{Na}$ ) and a heavy (the ${ }^{127} \mathrm{I}$ ) target-nucleus; in previous corollary quests WIMP masses above $30 \mathrm{GeV}$ (25 GeV in ref. [i] $\overline{1} \overline{1}]$ ) have been presented

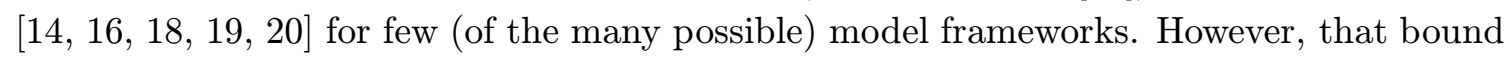
holds only for neutralino when supersymmetric schemes based on GUT assumptions are adopted to analyse the LEP data [2 $\left.2{ }_{1}^{4}\right]$. Thus, since other candidates are possible and also other scenarios can be considered for the neutralino itself as recently pointed out 6 , the present model dependent lower bound quoted by LEP for the neutralino in the supersymmetric schemes based on GUT assumptions (37 GeV [2] $\left.2 \overline{z_{1}}\right)$ is simply marked in the following figures. It is worth to note that when this mass limit is adopted, it selects the WIMP-Iodine elastic scattering as dominant because of the used scaling laws and of kinematical arguments. Finally, the prior from DAMA/NaI-0 has properly been considered as well.

WIMPs with mixed SI\&SD interaction. The most general scenario of WIMP nucleus elastic interaction, to which the DAMA/NaI target nuclei are fully sensitive, is the one

\footnotetext{
${ }^{6}$ In fact, when the assumption on the gaugino-mass unification at GUT scale is released neutralino

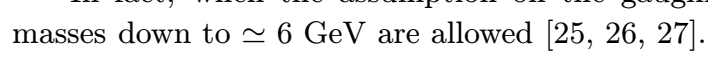


where both the SI and the SD components of the cross section are present. Thus, as first we introduce here the case for a candidate with both SI and SD couplings to ordinary

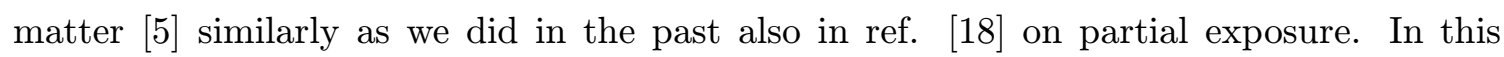
general scenario the space of the free parameters is a 4-dimensional volume defined by $m_{W}, \xi \sigma_{S I}{ }^{7}, \xi \sigma_{S D}$ and $\theta$ (which varies from 0 to $\pi$ ). Thus, the general solution would be a four dimensional allowed volume for each considered model framework. Since the graphic representation of this allowed volume is quite difficult, we only show in Fig. 崔 the obtained regions in the plane $\xi \sigma_{S I}$ vs $\xi \sigma_{S D}$ for some of the possible $\theta$ and $m_{W}$ values in the model frameworks considered here. In particular, we report just four couplings, which correspond to the following values of the mixing angle $\theta$ : i) $\theta=0\left(a_{n}=0\right.$ and $a_{p} \neq 0$ or $\left.\left|a_{p}\right|>>\left|a_{n}\right|\right)$ corresponding to a particle with null SD coupling to neutron; ii) $\theta=\pi / 4$ $\left(a_{p}=a_{n}\right)$ corresponding to a particle with the same SD coupling to neutron and proton; iii) $\theta=\pi / 2\left(a_{n} \neq 0\right.$ and $a_{p}=0$ or $\left.\left|a_{n}\right|>>\left|a_{p}\right|\right)$ corresponding to a particle with null SD couplings to proton; iv) $\theta=2.435 \mathrm{rad}\left(\frac{a_{n}}{a_{p}}=-0.85\right)$ corresponding to a particle with SD coupling through $Z_{0}$ exchange. The case $a_{p}=-a_{n}$ is nearly similar to the case iv).

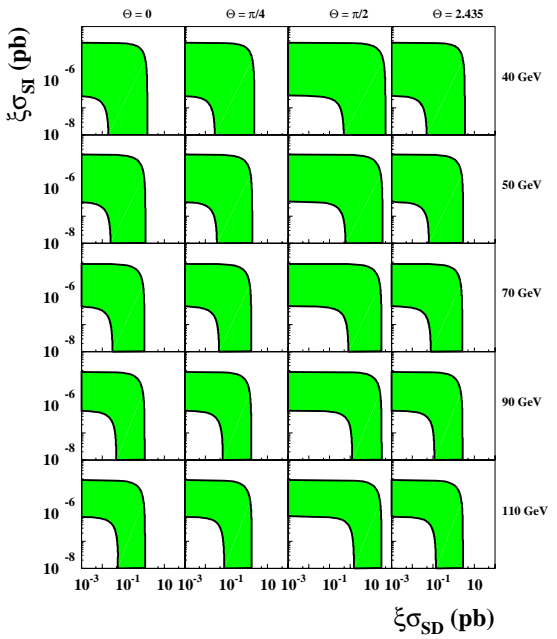

Figure 4: Case of a WIMP with mixed SIESSD interaction in the given model frameworks. Coloured areas: example of slices (of the allowed volume) in the plane $\xi \sigma_{S I}$ vs $\xi \sigma_{S D}$ for some of the possible $m_{W}$ and $\theta$ values. Inclusion of other existing uncertainties on parameters and models would further extend the regions; for example, the use of more favourable form factors and/or of more favourable spin factors than the considered ones would move them towards lower cross sections. For details see [i5i]l.
From the given figures it is clear that at present either a purely SI or a purely SD or a mixed SI\&SD configurations are supported by the experimental data of the seven annual cycles.

WIMPs with dominant SI interaction. Generally, the case of purely SI coupled WIMP is mainly considered in literature. In fact, often the spinindependent interaction with ordinary matter is assumed to be dominant since e.g. most of the used target-nuclei are practically not sensitive to SD interactions (as on the contrary ${ }^{23} \mathrm{Na}$ and ${ }^{127} \mathrm{I}$ are) and the theoretical calculations are even much more complex and uncertain.

Thus, following an analogous procedure as for the previous case, we have exploited for the same model frameworks the purely SI scenario. In this case the free parameters are two: $m_{W}$ and $\xi \sigma_{S I}$.

In Fig. $\overline{5_{1}}-$ left the region allowed in the plane $m_{W}$ and $\xi \sigma_{S I}$ for the considered model frameworks is reported. The configurations below the vertical line are of interest for neutralino when the assumption on the gaugino-mass unification at GUT scale is released and for every other kind of WIMP candidate. As shown in Fig. above $200 \mathrm{GeV}$ are allowed for some configurations;

details can be found in ref. [i5i]. Of course, best fit values of cross section and WIMP mass span over a large range in the considered frameworks.

\footnotetext{
${ }^{7} \xi(\xi \leq 1)$ is defined here as the fractional amount of local WIMP density.
} 

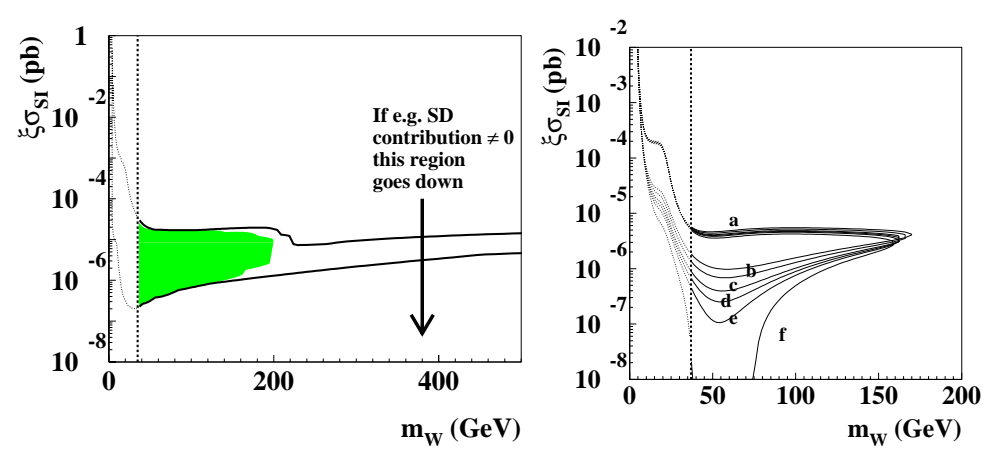

Figure 5: On the left: Case of a WIMP with dominant SI interaction for the given model frameworks. Region allowed in the plane $\left(m_{W}, \xi \sigma_{S I}\right)$. The vertical dotted line represents a bound in case of a neutralino candidate when supersymmetric schemes based on GUT assumptions are adopted to analyse the LEP data; the low mass region is allowed for neutralino when other schemes are considered and for every other WIMP candidate; see text. While the area at WIMP masses above $200 \mathrm{GeV}$ is allowed only for few configurations, the lower one is allowed by most configurations (the colored region gathers only those above the vertical line) $[\overline{5}|\bar{n}|$. The inclusion of other existing uncertainties on parameters and models would further extend the region; for example, the use of more favourable SI form factor for Iodine alone would move it towards lower cross sections. On the right: Example of the effect induced by the inclusion of a SD component different from zero on allowed regions given in the plane $\xi \sigma_{S I}$ vs $m_{W}$. In this example the Evans' logarithmic axisymmetric $C 2$ halo model with $v_{0}=170 \mathrm{~km} / \mathrm{s}, \rho_{0}$ equal to the maximum value for this model and a given set of the parameters' values (see [i, have been considered. The different regions refer to different SD contributions for the particular case of $\theta=0: \sigma_{S D}=0 \mathrm{pb}(\mathrm{a}), 0.02 \mathrm{pb}(\mathrm{b}), 0.04 \mathrm{pb}(\mathrm{c}), 0.05 \mathrm{pb}$ (d), $0.06 \mathrm{pb}(\mathrm{e}), 0.08 \mathrm{pb}$ (f). Analogous situation is found for the other model frameworks.

Let us now point out, in addition, that configurations with $\xi \sigma_{S I}$ even much lower than those shown in Fig. $5.5-1$ - left are accessible in case an even small SD contribution is present in the interaction. This possibility is clearly pointed out in Fig. 占- right where an example of allowed regions in the plane $\left(m_{W}, \xi \sigma_{S I}\right)$ corresponding to different SD contributions is reported for the case $\theta=0$. As it can be seen, increasing the SD contribution the allowed regions involve SI cross sections much lower than $10^{-6} \mathrm{pb}$; it can be noted that for $\sigma_{S D} \geq 0.08 \mathrm{pb}$ the annual modulation effect observed is also compatible - for $m_{W} \simeq 40-75$ $\mathrm{GeV}$ - with a WIMP candidate with no SI interaction at all. Analogous situation is found for the other model frameworks.

WIMPs with dominant SD interaction. Let us now focus on the case of a candidate with purely SD coupling to which DAMA/NaI is - as mentioned - fully sensitive.

When the SD component is different from zero, a very large number of possible configurations is available. In fact, in this scenario the space of free parameters is a 3 -dimensional volume defined by $m_{W}, \xi \sigma_{S D}$ and $\theta$ (which can vary from 0 to $\pi$ ). Here, just as an example we show the results obtained only for a particular coupling, which correspond to a mixing angle $\theta=2.435$ ( $Z_{0}$ coupling); see Fig. ' $6_{0}^{\mathrm{k}}$ other configurations are possible varying the $\theta$ value. The area at WIMP masses above $200 \mathrm{GeV}$ is allowed for low local velocity and all considered sets of parameters by the Evans' logarithmic $C 2$ co-rotating halo model Moreover, the accounting for the uncertainties e.g. on the form factors and/or on the spin 
factors as well as different possible formulations of the SD form factors would extend the allowed regions, e.g. towards lower $\xi \sigma_{S D}$ values. Finally, $\xi \sigma_{S D}$ lower than those corresponding to the regions shown in Fig. ' ${ }_{-1}^{\prime} \bar{i}_{1}^{\prime}$ are possible also e.g. in case of an even small SI contribution (see ref. $[\dot{\bar{p}}|\bar{i}|)$.

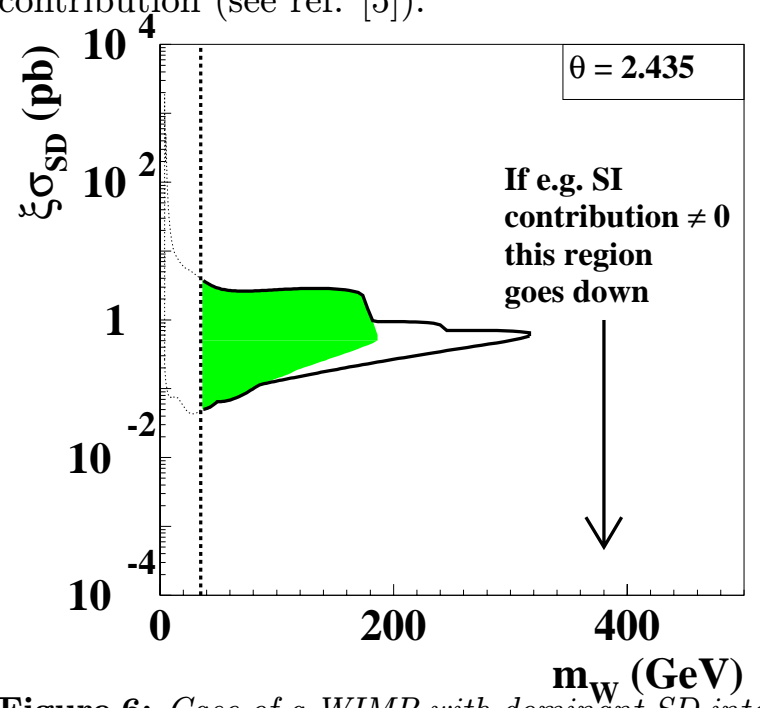

Figure 6: Case of a WIMP with dominant SD interaction in the given model frameworks. An example of the region allowed in the plane $\left(m_{W}, \xi \sigma_{S D}\right)$; here $\theta=2.435, Z_{0}$ coupling, $(\theta$ is defined in the $[0, \pi)$ range). For the definition of the vertical line and of the coloured area see previous figure caption; see also text. Inclusion of other existing uncertainties on parameters and models (as discussed in ref. [i5i]) would further extend the SD allowed regions. For example, the use of more favourable SD form factors and/or more favourable spin factors would move them towards lower cross sections. Values of $\xi \sigma_{S D}$ lower than those corresponding to these allowed regions are possible also e.g. in case of an even small SI contribution (see ref. $[\underline{\tilde{s}} \bar{\eta}]$ ).

\section{WIMPs with preferred inelastic in-} teraction. An analysis considering the same model frameworks has been carried out for the case of WIMPs with preferred inelastic interaction [i] $\left.{ }_{1}^{1} \overline{0}\right]$ as we did also in the past in ref. [i] $\left.{ }^{1} \bar{g}\right]$ on partial exposure.

In this inelastic Dark Matter scenario an allowed volume in the space $\left(\xi \sigma_{p}, m_{W}, \delta\right)$ is obtained; $\delta$ is the mass splitting of the WIMP particle which can be excited following an inelastic interaction [1] 100 i'i'shows slices of such an allowed volume at some given WIMP masses. There the superpositions of the allowed regions obtained, when varying the model framework within the considered set [5], are shown for each $m_{W}$. We remind that in these calculations $v_{e s c}$ has been assumed at fixed value (as in the previous cases), while its present uncertainties can play a significant role in this scenario of WIMP with preferred inelastic scattering.

Conclusion. In this section we have briefly summarized some quests for the candidate particle in some of the many possible scenarios. We further stress that, although several scenarios have been investigated, these corollary analyses are not exhaustive at all because of the present poor knowledge on many astrophysical, nuclear and particle Physics needed assumptions; moreover, additional scenarios can also be possible as also shown e.g. by some recent papers appeared in literature. Other model dependent quests are already under consideration.

\section{The second generation: DAMA/LIBRA}

In 1996 a ton set-up was proposed by Bernabei et al.; as a consequence a new R\&D for the $\mathrm{NaI}(\mathrm{Tl})$ radiopurification has been carried out and the second generation set-up DAMA/LIBRA $(\simeq 250 \mathrm{~kg} \mathrm{NaI}(\mathrm{Tl}))$ has been funded and realised as an intermediate step. 
This R\&D with Crismatec-St. Gobain company has exploited new chemical/physical radiopurification procedures in NaI and TII powders further selected for radiopurity. In addition, new selected materials and set-up components as well as new protocols have been employed for building, handling and installing DAMA/LIBRA. After July 2002 - at the completion of its data taking - DAMA/NaI was fully dismounted and the installation of the new DAMA/LIBRA started. The experimental site as well as many components of the installation itself were implemented. In particular, all the procedures performed during the dismounting of DAMA/NaI and the installation of DAMA/LIBRA were carried out in HP Nitrogen atmosphere.

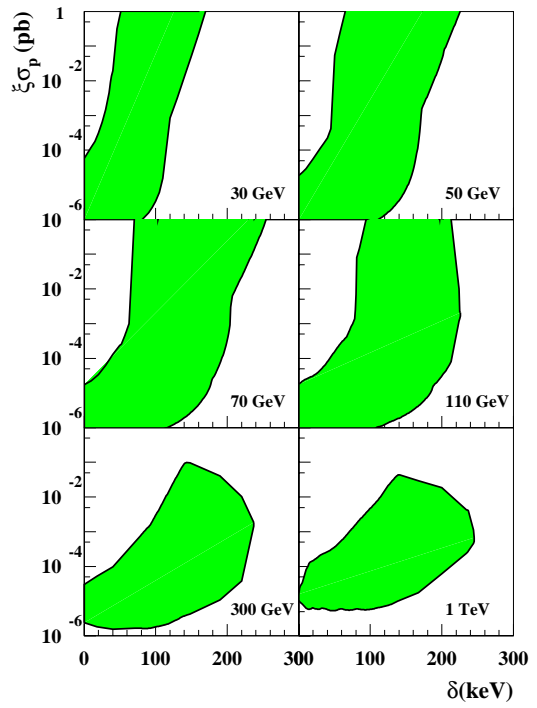

Figure 7: Case of a WIMP with preferred inelastic interaction in the given model frameworks. Examples of slices (coloured areas) of the allowed volumes $\left(\xi \sigma_{p}, \delta, m_{W}\right)$ for some $m_{W}$ values for WIMP with preferred inelastic interaction. Inclusion of other existing uncertainties on parameters and models would further extend the regions [15]1].

The new DAMA/LIBRA, having an higher mass, overall radiopurity and improved performances, offers an increased experimental sensitivity to further investigate the DAMA/NaI observed effect and to improve investigations on the nature of the candidate particle trying to disentangle among different possible astrophysical, nuclear and particle Physics models as well as other new possible scenarios such as e.g. those reported in refs. $[\overline{1} \overline{1} \overline{1}, 1, \overline{2} 2 \overline{2}, 1, \overline{2} \overline{3}]$. In particular, the low background DAMA/LIBRA offers a powerful tool for the Dark Matter investigation in the future since it is e.g. sensitive: i) both to low (through interaction on ${ }^{23} \mathrm{Na}$ ) and to high (through interaction on ${ }^{127} \mathrm{I}$ ) mass Dark Matter particles; ii) both to mixed SI\&SD, to purely SI, to purely SD couplings and to preferred inelastic scattering as well as to other possible kind of Dark Matter candidates (e.g. mirror Dark Matter).

DAMA/LIBRA has started the preliminary data taking on March 2003 and it has been planned to run for several years. At present a new R\&D effort toward the possible $\mathrm{NaI}(\mathrm{Tl})$ ton set-up has been funded and related works have already been started.

\section{References}

[1] P. Belli, R. Bernabei, C. Bacci, A. Incicchitti, R. Marcovaldi, D. Prosperi, DAMA proposal to INFN Scientific Committee II, April $24^{\text {th }} 1990$

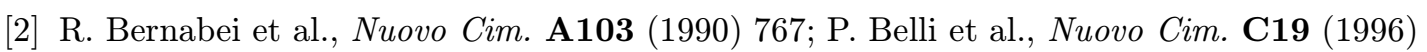
- $53 \overline{7}_{;}$P. Belli et al., Astropart. Phys.5 1996$) 217_{;}$P. Belli et al., $==222$ and 'Phys.Lett. B $389(1996) 783$ (erratum); R. Bernabei et al., ,Phys. Lett. B 436 - $493(2000) 12$ R. Bernabei et al., New Journal of Physics 2 (2000) 15.1; P. Belli et al., iPhys.'

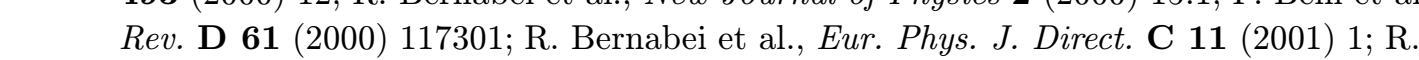

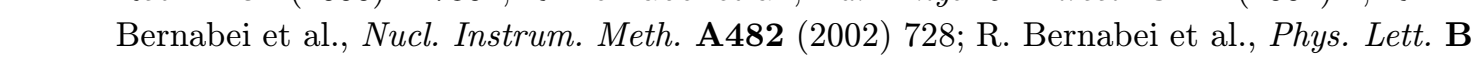

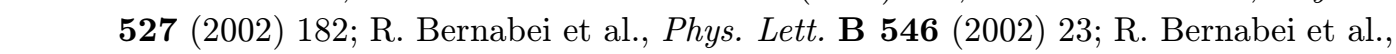


LNGS/EXP-08/03 (available at www.lngs.infn.it), to appear on the Proc. of "Beyond the Desert 03", Rindberg Castle, June 2003

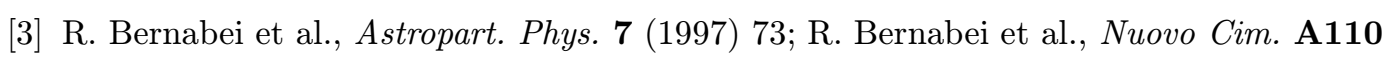
; (1999)115.; R. Bernabei et al., 'Nucl. Phys. A 705.2002)29; P. Belli et al., Nochl. Instrum. Meth. A 498 - 2003$) 352$

[4] R. Bernabei et al., 'Nuovo Cim. Ā12 (1999) $54 \overline{5}$

[5] R. Bernabei et al., La Rivista del Nuovo Cimento 26 (2003) 1-73 [astro-ph/0307403]

[6] R. Bernabei et al., iPhys. Lett. B $\mathbf{3} \mathbf{3 9} 9(1996) 75 \overline{7}$,

[7] R. Bernabei et al., Nuovo Cim. Āî12

[8] R. Bernabei et al., 'Phys. Rev. Lett. 83 (1999) 4918; F. Cappella et al., Eur. Phys. J. Direct! C $14(200 \overline{2}) \overline{1} 1$ R. Bernabei et al.,

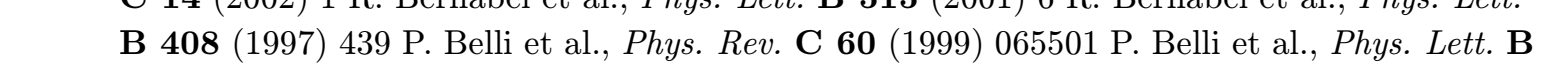
-

[9] K.A. Drukier et al., 'L

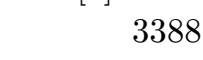

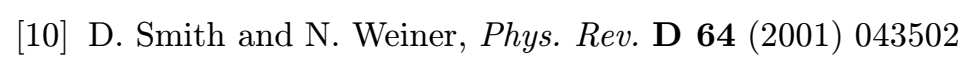

[11] K. Freese et al., astro-ph/0309279

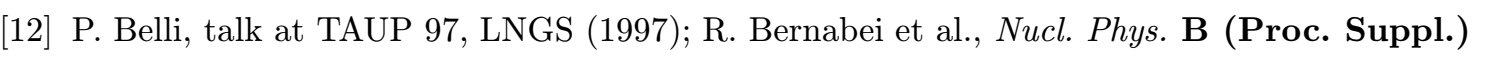
(19)

[13] R. Bernabei et al., Phys. Lett. B

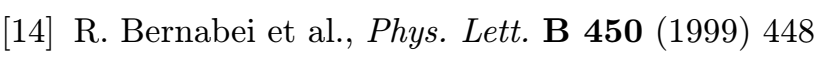

[15] P. Belli et al., 'P

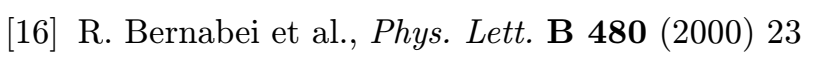

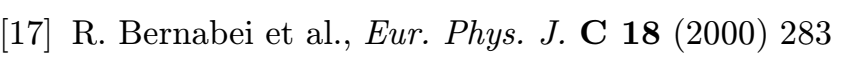

[18] R. Bernabei et al., 'Phys. Lett. B $\mathbf{5} 0 \mathbf{0 9}(2001) 19 \bar{q}$

[19] R. Bernabei el al., Eur. Phys. J.

[20] P. Belli et al., 'Phys. Rev. D- $6 \mathbf{6} 6(2002) 043503$

[21] W.H. Press and G. B. Rybicki, 'Ástrophys. (1982) $83 \overline{5}$

[22] R. Foot, astro-ph/0309330, hep-ph/0308254

[23] G. Prezeau et al., astro-ph/0309115

[24] D.E. Groom et al., 'Eur. Phys. J.

[25] A. Bottino et al., 'P

[26] D. Hooper and T. Plehn, MADPH-02-1308, CERN-TH/2002-29, [hep-ph/0212226]

[27] G. Bélanger, F. Boudjema, A. Pukhov and S. Rosier-Lees, hep-ph/0212227

[28] K. Hagiwara et al., 'Phys. Rev. D 66 6 20022010001 